\title{
Synthesis, structure, and antiviral properties of novel 2-adamantyl-5-aryl-2H-tetrazoles
}

\author{
Olga V. Mikolaichuk ${ }^{1}$, Vladimir V. Zarubaev ${ }^{2}$, Anna A. Muryleva', \\ Yana L. Esaulkova ${ }^{2}$, Daria V. Spasibenko ${ }^{3}$, Alina A. Batyrenko ${ }^{3}$, \\ Ilya V. Kornyakov ${ }^{1,4}$, Rostislav E. Trifonov ${ }^{3 *}$ \\ ${ }^{1}$ Saint Petersburg State University, \\ 7/9 University Embankment, Saint Petersburg 199034, Russia; e-mail: st801293@spbu.ru \\ ${ }^{2}$ Saint Petersburg Pasteur Research Institute of Epidemiology and Microbiology, \\ 14 Mira St., Saint Petersburg 197101,Russia; e-mail: zarubaev@gmail.com \\ ${ }^{3}$ Saint Petersburg State Institute of Technology (Technical University), \\ 26 Moskovsky Ave., Saint Petersburg 190013, Russia; e-mail: rost_trifonov@mail.ru \\ ${ }^{4}$ Kola Science Center, Russian Academy of Sciences, \\ 14 Fersmana St., Apatity 184209, Murmansk Oblast, Russia; e-mail: i.kornyakov@spbu.ru
}

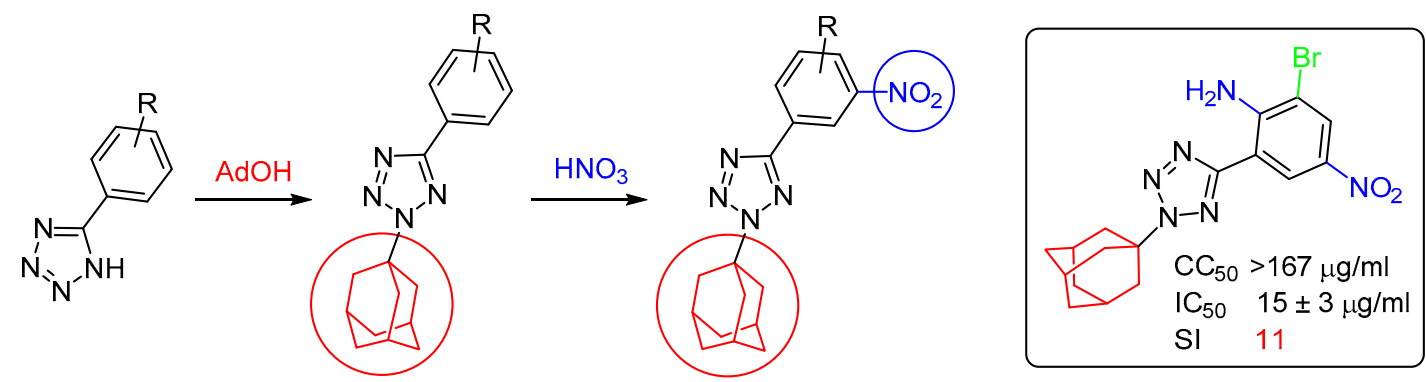

The reaction of 5-aryl- $\mathrm{NH}$-tetrazoles with adamantan-1-ol in concentrated sulfuric acid proceeds regioselectively with the formation of the corresponding 2-adamantyl-5-aryl-2H-tetrazoles. Nitration of these compounds leads to 2-(adamantan-1-yl)-5-(3-nitroaryl)-2Htetrazoles. The structures and composition of the obtained novel 2-adamantyl-5-aryltetrazoles were proven by IR spectroscopy, ${ }^{1} \mathrm{H}$ and ${ }^{13} \mathrm{C}$ NMR spectroscopy, high-resolution mass spectrometry, and also by X-ray structural analysis. According to the simultaneous thermal analysis data, the obtained compounds are thermally stable up to a temperature of about $150^{\circ} \mathrm{C}$. In vitro studies have shown that some of the 2-adamantyl-5-aryltetrazoles exhibit moderate inhibitory activity against influenza A (H1N1) virus. The antiviral selectivity index (SI) of 2-[2-(adamantan-1-yl)-2H-tetrazol-5-yl]-6-bromo-4-nitroaniline is significantly higher (SI 11) than that of the reference drug rimantadine (SI 5).

Keywords: 2-(adamantan-1-yl)-5-aryl-2H-tetrazoles, adamantylation, anti-influenza activity, nitration, X-ray structural analysis.

It is known that a number of adamantane derivatives and other cage hydrocarbons exhibit high antiviral activity. ${ }^{1}$ Some of these compounds are known drugs against influenza virus (amantadine, rimantadine). ${ }^{4}$ (Adamantan-2-yl)1-(2-oxoindolin-3-ylidene)thiosemicarbazide A (Fig. 1) reduces the reproducibility of vaccinia virus, and also exhibits activity in vitro against some Gram-positive and Gramnegative bacteria and fungi. ${ }^{2,3}$ Substituted aminoacetyladamantylamines $\mathbf{B}$ exhibit inhibitory activity against vaccinia virus (Fig. 1). ${ }^{3}$ 1-Adamantanecarboxylic acid amide containing the (-)-10-amino-2-pinene fragment (structure C) ${ }^{4}$ and $N-(1-$ adamantyl)-2,2-diphenylacetamide $\mathbf{D}$ proved to be effective inhibitors of smallpox virus. ${ }^{5}$ In addition, compounds containing the adamantyl fragment such as memantine $\mathbf{E}$ (Fig. 1) are also capable of inhibiting the $N$-methyl-D-aspartate receptor, which allows them to be considered as potential agents for the treatment of moderate and severe Alzheimer's disease. ${ }^{6}$ Adamantane derivatives have shown in vitro activity against various coronaviruses. ${ }^{7,8}$ For instance, amantadine analogs are moderately active against human coronavirus $229 E^{7}{ }^{7}$ Moderate antiviral activity has also been found for amantadine, rimantadine, memantine in bovine models. ${ }^{8}$ 
<smiles>O=C1Nc2ccccc2/C1=N\NC(=S)NC1C2CC3CC(C2)CC1C3</smiles><smiles>O=C(CNC1CCCCC1)NC12CC3CC(CC(C3)C1)C2</smiles><smiles>CC12CC3(C)CC(N)(C1)CC(C)(C3)C(C)(C)C2</smiles>

Figure 1. Adamantane-based biologically active compounds.

One of the promising design options for new highly effective anti-influenza drugs is the replacement of the amino group with polynitrogen heterocyclic fragments. ${ }^{9-11}$ This fragment can be the tetrazole ring, a well-known pharmacophore $^{9-11}$ due to its high stability in metabolic processes and low toxicity. Tetrazole derivatives containing the adamantyl moiety showed significant activity against influenza A virus, including rimantadine-resistant influenza virus strain A/Puerto Rico/8/34 (Fig. 2). ${ }^{12,13}$

In this work, we obtained a series of $\mathrm{NH}$-unsubstituted 5 -aryltetrazoles $\mathbf{2 a}-\mathbf{h}$ by azidation of aromatic nitriles $\mathbf{1 a}-\mathbf{h}$ via the 1,3-dipolar cycloaddition reaction (Scheme 1). Azidation was carried out in DMF according to a known method at $115^{\circ} \mathrm{C} .{ }^{11-14}$ The reaction of 5-aryl- $\mathrm{NH}$-tetrazoles 2a-h with adamantan-1-ol in concentrated $\mathrm{H}_{2} \mathrm{SO}_{4}$ gave a series of novel 2-adamantyl-5-aryl- $2 \mathrm{H}$-tetrazoles $3 \mathbf{a}-\mathbf{h}$. The adamantylation of $\mathrm{NH}$-tetrazoles $\mathbf{2} \mathbf{a}-\mathbf{h}$ in $\mathrm{H}_{2} \mathrm{SO}_{4}$ proceeds exclusively at the $\mathrm{N}-2$ nitrogen atom of the tetrazole ring with the formation of 2-(adamantan-1-yl)-5-aryl-2Htetrazoles 3a-h in high (81-92\%) yields. Compounds $\mathbf{3 c , d}$ were nitrated with a sulfuric-nitric acid mixture at $8-10^{\circ} \mathrm{C}$ according to the previously described method ${ }^{15}$ to give 2-(adamantan-1-yl)-5-(nitroaryl)- $2 H$-tetrazoles $\mathbf{3 j}, \mathbf{k}$. Compound $\mathbf{3 i}$ was obtained by reduction of nitro compound $\mathbf{3} \mathbf{g}$ with metallic zinc.

The structure, composition, and thermal stability of the obtained compounds were studied by IR spectroscopy, ${ }^{1} \mathrm{H}$, ${ }^{13} \mathrm{C}$ NMR spectroscopy, high-resolution mass spectrometry, $\mathrm{X}$-ray structural analysis, and simultaneous thermal analysis. The ${ }^{1} \mathrm{H}$ and ${ }^{13} \mathrm{C}$ NMR spectra confirm the structures of all the obtained compounds. In the ${ }^{1} \mathrm{H}$ NMR
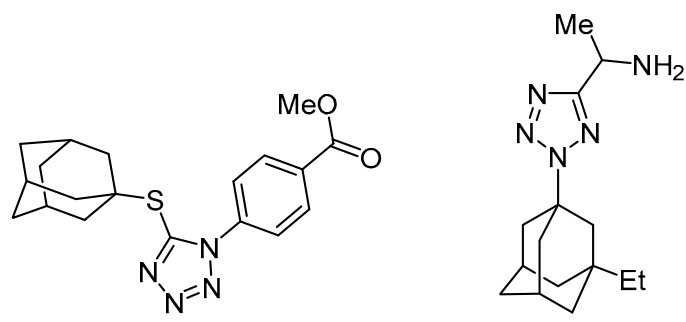

Figure 2. Adamantyltetrazoles showing high inhibitory activity against influenza virus.
Scheme 1
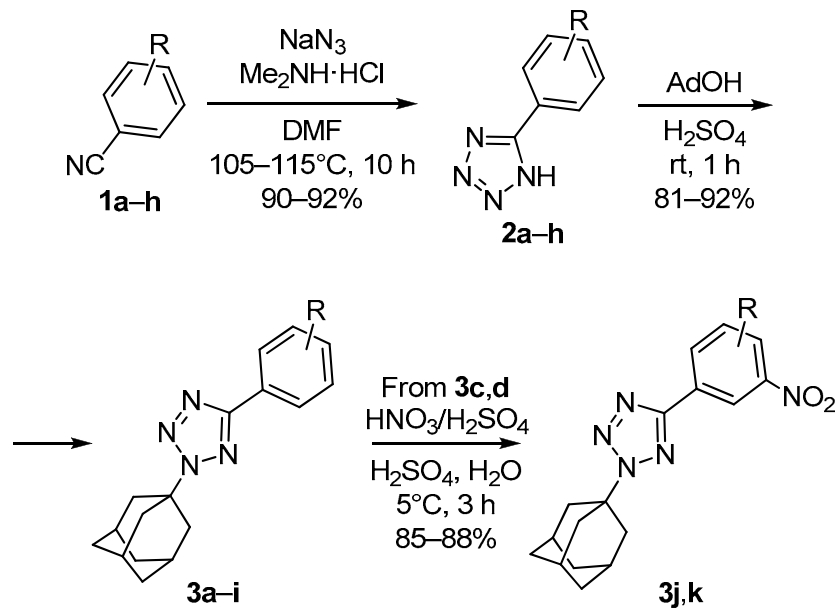

a $\mathrm{R}=4-\mathrm{l} ; \mathbf{b} \mathrm{R}=2-\mathrm{Me} ; \mathbf{c} \mathrm{R}=3-\mathrm{NO}_{2} ; \mathbf{d} \mathrm{R}=4-\mathrm{Cl} ; \mathbf{e ~ R}=3-\mathrm{Cl}, 4-\mathrm{NH}_{2}$; f R = 2-Cl, 4-CF $;$, g R = 3-NO $\mathrm{NO}_{2}, 4-\mathrm{OH} ; \mathbf{h ~ R}=2-\mathrm{NH}_{2}, 3-\mathrm{Br}, 5-\mathrm{NO}_{2}$; i R = 3- $\mathrm{NH}_{2}, 4-\mathrm{OH} ; \mathbf{j} \mathrm{R}=3,5-\left(\mathrm{NO}_{2}\right)_{2} ; \mathbf{k ~ R}=3-\mathrm{NO}_{2}, 4-\mathrm{Cl}$

spectra of 2-(adamantan-1-yl)-5-aryl-2H-tetrazoles $\mathbf{3 a}-\mathbf{k}$, the adamantyl moiety appears as broadened singlets at $1.76-$ 1.87 (compounds 3a-g,i-k), 2.16 (compound 3h), and 2.22$2.36 \mathrm{ppm}$ and in the form of broadened singlets or multiplets in the $2.29-2.43 \mathrm{ppm}$ range. In the ${ }^{13} \mathrm{C}$ NMR spectra of compounds $\mathbf{3 a}-\mathbf{k}$, the signals of the endocyclic carbon atom of the tetrazole fragment were observed at 161.1-164.9 ppm which reliably confirms the preparation of $2 \mathrm{H}$-regioisomers. ${ }^{14}$

The IR spectra of compounds $\mathbf{3 a}, \mathbf{b}, \mathbf{e}, \mathbf{h}$ contain the characteristic absorption bands of the cage hydrocarbon group in the range of $3346-2850 \mathrm{~cm}^{-1}$ which is consistent with previously published data. ${ }^{16}$

The data of X-ray structural analysis of compounds $\mathbf{3 b}, \mathbf{e}-\mathbf{g}$ also unambiguously confirm the structure of the obtained compounds (Fig. 3). According to these data, the $2 \mathrm{H}$-tetrazole ring is a planar highly aromatic system. The dihedral angle between the planes of the benzene and tetrazole rings in compounds $\mathbf{3 b}, \mathbf{e}-\mathbf{g}$ is small, which may indicate $\pi-\pi$ conjugation between unsaturated fragments. In all of the studied compounds, the tetrazole ring appears to be partially sterically blocked by a cage hydrocarbon fragment.

It is known that some $2 H$-tetrazoles, especially those containing electron-withdrawing substituents, can relatively easily undergo thermal decomposition with the dissociation of the $\mathrm{N}-\mathrm{N}$ bonds and release of molecular nitrogen. ${ }^{11,14}$ The thermal stability of compound $\mathbf{3 g}$ was studied by the method of simultaneous thermal analysis (Fig. S54, Supplementary information file). This compound contains an electron-withdrawing nitro group in the aryl moiety and is a good model for assessing the stability of compounds of this series. According to the loss of weight, in the case of compound $3 \mathrm{~g}$ molecular nitrogen is released at $156.2^{\circ} \mathrm{C}$. This process is accompanied by a strong exothermic effect. The complete decomposition of adamantyltetrazole $\mathbf{3 g}$ occurs starting with $250^{\circ} \mathrm{C}$ with a loss of $33.6 \%$ of its mass.

In this work, we studied the antiviral activity of adamantyltetrazole derivatives $\mathbf{3 a}, \mathbf{b}, \mathbf{d}, \mathbf{e}, \mathbf{h ,}, \mathbf{i}$ against influenza 

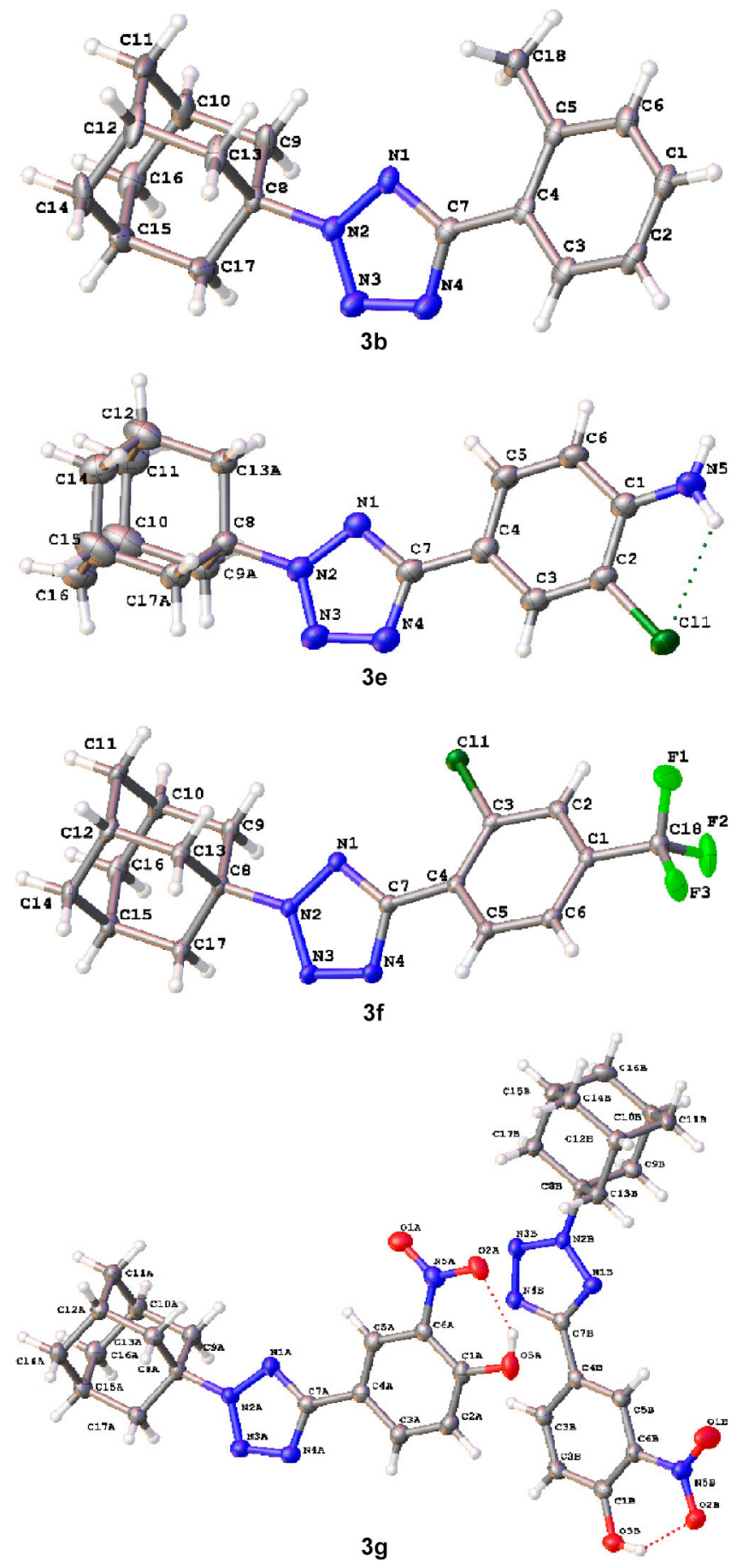

Figure 3. Molecular structure of compounds $\mathbf{3 b}, \mathbf{e}, \mathbf{f}, \mathbf{g}$ with atoms represented as thermal vibration ellipsoids of $50 \%$ probability.

A/Puerto Rico/8/34 (H1N1) virus (Table 1). Analysis of the obtained data shows that compounds 3a,d,e containing a halogen atom as a substituent mainly showed moderate inhibitory activity against the virus $\left(\mathrm{IC}_{50} 31-2 \mu \mathrm{g} / \mathrm{ml}\right)$ and rather high toxicity against cells $\left(\mathrm{CC}_{50} 46-4 \mu \mathrm{g} / \mathrm{ml}\right)$, which is expressed by the low values of the selectivity index (SI). At the same time, tetrazole derivative $\mathbf{3 h}$ containing an amino group and a bromine atom turned out to be the least toxic to cells $\left(\mathrm{CC}_{50} 167 \mu \mathrm{g} / \mathrm{ml}\right)$ in combination with
Table 1. Cytotoxic $\left(\mathrm{CC}_{50}\right)$, antiviral $\left(\mathrm{IC}_{50}\right)$ activity, selectivity index (SI) of adamantyltetrazole derivatives $\mathbf{3 a}, \mathbf{b}, \mathbf{d}, \mathbf{e}, \mathbf{h}, \mathbf{i}$ against the $\mathrm{H} 1 \mathrm{~N} 1$ influenza A virus strain

\begin{tabular}{cccc}
\hline Compound & $\mathrm{CC}_{50}, \mu \mathrm{g} / \mathrm{ml}$ & $\mathrm{IC}_{50}, \mu \mathrm{g} / \mathrm{ml}$ & $\mathrm{SI}$ \\
\hline 3a & $46 \pm 3$ & $31 \pm 4$ & 1 \\
3b & $>300$ & $>300$ & 1 \\
3d & $4 \pm 0.5$ & $2 \pm 0.3$ & 2 \\
3e & $<3$ & $<3$ & - \\
3h & $>167$ & $15 \pm 3$ & 11 \\
3i & $36 \pm 2$ & $15 \pm 2$ & 2 \\
Rimantadine & $60 \pm 4$ & $12 \pm 2$ & 5 \\
\hline
\end{tabular}

moderate activity against the virus $\left(\mathrm{IC}_{50} 15 \mu \mathrm{g} / \mathrm{ml}\right)$, which is expressed by the relatively high value of the selectivity index of SI 11 exceeding the SI value of the reference drug rimantadine.

The influenza virus used in the study shows resistance to rimantadine due to the $\mathrm{S} 31 \mathrm{~N}$ mutation in the viral protein M2. The activity of compound $\mathbf{3 h}$ demonstrated against this virus may indicate both that it is a specific inhibitor of such a mutant ion channel and that the target of its action is another viral protein and the mechanism of action of compounds of this class is not related to blocking the M2 proton channel.

To conclude, employing the regioselective alkylation of 5-aryl- $\mathrm{NH}$-tetrazoles with adamantan-1-ol in sulfuric acid, a series of new 2-adamantyl-5-aryl-2 $H$-tetrazoles were synthesized. The structure of the obtained compounds was proved by spectral methods and by the X-ray structural analysis technique. Some of the 2-adamantyl-5-aryltetrazoles exhibit moderate inhibitory activity against influenza A (H1N1) virus and good selectivity. 2-Adamantyl5 -aryl- $2 H$-tetrazoles are promising compounds which can display high anti-influenza activity.

\section{Experimental}

IR spectra were registered on an IRPrestige-21 Fourier transform spectrometer in $\mathrm{KBr}$ pellets. ${ }^{1} \mathrm{H}$ and ${ }^{13} \mathrm{C}$ NMR spectra were acquired on a Bruker Avance III 400 spectrometer (400 and $100 \mathrm{MHz}$, respectively) at $25^{\circ} \mathrm{C}$. High-resolution mass spectrometry was performed on a Bruker MicroTOF spectrometer (electrospray ionization). Melting points were determined on a PTP apparatus using $1{ }^{\circ} \mathrm{C} /$ min heating rate in the melting range. Simultaneous thermal analysis was performed on a Netzsch STA 449F1 apparatus under a flow of $\operatorname{argon}\left(100 \mathrm{ml} / \mathrm{min}^{-1}\right)$ with a heating rate of $10^{\circ} \mathrm{C} / \mathrm{min}$ in aluminum crucibles. TLC was performed on Merck KGaA $60 \mathrm{~F}_{254}$ plates, eluent $\mathrm{CHCl}_{3}$ $\mathrm{MeOH}, 19: 1$.

Synthesis of 5-aryl- $\mathrm{NH}$-tetrazoles 2a-h (General method). The corresponding nitrile $\mathbf{1 a}-\mathbf{h}(10 \mathrm{mmol})$ was added to a solution of $\mathrm{NaN}_{3}(11.4 \mathrm{mmol})$ and $\mathrm{Me}_{2} \mathrm{NH} \cdot \mathrm{HCl}$ $(11.2 \mathrm{mmol})$ in DMF $(40 \mathrm{ml})$. The resulting suspension was heated at $105-115^{\circ} \mathrm{C}$ for $10 \mathrm{~h}$. Then, the reaction mixture was cooled, the precipitate was filtered off and additionally 
washed with DMF $(2 \times 5 \mathrm{ml})$. Then cold $\mathrm{H}_{2} \mathrm{O}(50 \mathrm{ml})$ was added to the filtrate and acidified with a solution of $10 \%$ $\mathrm{HCl}$ to $\mathrm{pH} 2-3$. The resulting suspension was filtered, and the precipitate was washed with cold $\mathrm{H}_{2} \mathrm{O}$. The product was air-dried. The physical and spectral characteristics of the obtained and previously described compounds $\mathbf{2 a - d , h}$ correspond to the literature. ${ }^{13,15,17}$

2-Chloro-4-(1H-tetrazol-5-yl)aniline (2e). Yield $1.76 \mathrm{~g}$ (90\%), white crystals, mp $169-170^{\circ} \mathrm{C} .{ }^{1} \mathrm{H}$ NMR spectrum $\left(\mathrm{DMSO}-d_{6}\right), \delta, \operatorname{ppm}(J, \mathrm{~Hz}): 6.05\left(2 \mathrm{H}, \mathrm{s}, \mathrm{NH}_{2}\right) ; 6.94(1 \mathrm{H}$, d, $J=8.5, \mathrm{H} \mathrm{Ar}) ; 7.71$ (1H, dd, $J=8.5, J=2.0, \mathrm{H} \mathrm{Ar}) ; 7.88$ $(1 \mathrm{H}, \mathrm{d}, J=2.0, \mathrm{H} \mathrm{Ar}){ }^{13} \mathrm{C}$ NMR spectrum (DMSO- $\left.d_{6}\right)$, $\delta$, ppm: 112.2, 115.8, 117.4, 127.3, 128.3, 147.9 (C Ar); 156.1 (C tetrazole). Found, $m / z: 196.0379 \quad[\mathrm{M}+\mathrm{H}]^{+}$. $\mathrm{C}_{7} \mathrm{H}_{7} \mathrm{ClN}_{5}$. Calculated, $m / z$ : 196.0385 .

5-[2-Chloro-4-(trifluoromethyl)phenyl]-1H-tetrazole (2f). Yield $2.29 \mathrm{~g}(92 \%)$, beige crystals, $\mathrm{mp} 179-181^{\circ} \mathrm{C}$. ${ }^{1} \mathrm{H}$ NMR spectrum $\left(\mathrm{DMSO}-d_{6}\right), \delta$, ppm $(J, \mathrm{~Hz}): 7.72(1 \mathrm{H}$, $\mathrm{dd}, J=8.2, J=1.8, \mathrm{H} \mathrm{Ar}) ; 7.86(1 \mathrm{H}, \mathrm{s}, \mathrm{H} \mathrm{Ar}) ; 8.07(1 \mathrm{H}, \mathrm{d}$, $J=8.2, \mathrm{H} \mathrm{Ar}$ ). ${ }^{13} \mathrm{C}$ NMR spectrum (DMSO- $\left.d_{6}\right), \delta$, ppm $(J, \mathrm{~Hz}): 122.6\left(\mathrm{~d}, J=270.4, \mathrm{CF}_{3}\right) ; 124.0,125.4,127.4$, 132.1 (2C), 136.4 (C Ar); 158.8 (C tetrazole). Found, $m / z$ : $270.9969 \quad[\mathrm{M}+\mathrm{Na}]^{+} . \quad \mathrm{C}_{8} \mathrm{H}_{4} \mathrm{ClF}_{3} \mathrm{~N}_{4} \mathrm{Na}$. Calculated, $m / z$ : 270.9969

2-Nitro-4-(1H-tetrazol-5-yl)phenol (2g). Yield $1.88 \mathrm{~g}$ (91\%), yellow crystals, mp $179-181{ }^{\circ} \mathrm{C} .{ }^{1} \mathrm{H}$ NMR spectrum $\left(\mathrm{DMSO}-d_{6}\right), \delta, \operatorname{ppm}(J, \mathrm{~Hz}): 7.34(1 \mathrm{H}, \mathrm{d}, J=8.7, \mathrm{H} \mathrm{Ar})$; $8.18(1 \mathrm{H}, \mathrm{dd}, J=8.7, J=2.3, \mathrm{H} \mathrm{Ar}) ; 8.55(1 \mathrm{H}, \mathrm{d}, J=2.2$, $\mathrm{H} \mathrm{Ar}) ; 11.84\left(1 \mathrm{H}\right.$, br. s, OH) ${ }^{13} \mathrm{C}$ NMR spectrum (DMSO- $d_{6}$ ), $\delta$, ppm: $120.8,124.6,133.7,137.6,138.3,154.6$ (C Ar); 155.8 (C tetrazole). Found, $m / z$ : $206.0318 \quad[\mathrm{M}-\mathrm{H}]^{-}$. $\mathrm{C}_{7} \mathrm{H}_{4} \mathrm{~N}_{5} \mathrm{O}_{3}$. Calculated, $m / z$ : 206.0309.

Synthesis of 2-(adamantan-1-yl)-5-aryl-2H-tetrazoles 3a-h (General method). Adamantan-1-ol (1.37 g, 9 mmol) was added to a solution of 5-arylterazole $\mathbf{2 a}-\mathbf{h}(9 \mathrm{mmol})$ in $94 \% \mathrm{H}_{2} \mathrm{SO}_{4}(25 \mathrm{ml})$. The reaction mixture was kept at room temperature for $60 \mathrm{~min}$ and poured into $\mathrm{H}_{2} \mathrm{O}$ /ice $(60 \mathrm{ml})$. The product was extracted with $\mathrm{CHCl}_{3}$, the organic phase was washed with $5 \%$ aqueous $\mathrm{NaHCO}_{3}$ $(2 \times 25 \mathrm{ml})$ followed by $\mathrm{H}_{2} \mathrm{O}$. It was then dried over anhydrous $\mathrm{MgSO}_{4}$ and evaporated under reduced pressure. The physical and spectral characteristics of the obtained and previously described compounds $\mathbf{3 b}-\mathbf{d}$ correspond to the literature. ${ }^{13,15}$

2-(Adamantan-1-yl)-5-(4-iodophenyl)-2H-tetrazole (3a). Yield $3.22 \mathrm{~g}(88 \%)$, light-orange crystals, $\mathrm{mp} 118-120^{\circ} \mathrm{C}$. IR spectrum, $v, \mathrm{~cm}^{-1}: 3295,2917,2850,1697,1449,1353$, $1302,1116,1088,927 .{ }^{1} \mathrm{H}$ NMR spectrum $\left(\mathrm{CDCl}_{3}\right), \delta$, ppm $(J, \mathrm{~Hz}): 1.85$ (6H, br. s, H Ad); 2.33 (3H, br. s, H Ad); 2.41 $(6 \mathrm{H}$, br. s, H Ad); 7.47 (2H, d, $J=8.4, \mathrm{H} \mathrm{Ar}) ; 8.12(2 \mathrm{H}, \mathrm{d}$, $J=8.8, \mathrm{H} \mathrm{Ar}) .{ }^{13} \mathrm{C}$ NMR spectrum $\left(\mathrm{CDCl}_{3}\right), \delta$, ppm: 29.7 , 36.1, 42.3, 64.9 (C Ad); 127.5, 128.4, 137.6, 138.0 (C Ar); 163.5 (C tetrazole). Found, $m / z: 429.0545[\mathrm{M}+\mathrm{Na}]^{+}$. $\mathrm{C}_{17} \mathrm{H}_{19} \mathrm{IN}_{4} \mathrm{Na}$. Calculated, $m / z$ : 429.0547.

2-(Adamantan-1-yl)-5-(o-tolyl)-2H-tetrazole (3b). Yield 2.44 g $(92 \%)$, light-beige crystals, mp $85-87^{\circ} \mathrm{C}(\mathrm{mp} 84-$ $86^{\circ} \mathrm{C}^{13}$ ). IR spectrum, $v, \mathrm{~cm}^{-1}: 3292,2924,2911,2856$, 1476, 1453, 1356, 1316, 1021, 746. ${ }^{1} \mathrm{H}$ NMR spectrum $\left(\mathrm{DMSO}-d_{6}\right), \delta, \mathrm{ppm}(J, \mathrm{~Hz}): 1.77(6 \mathrm{H}$, br. s, H Ad); 2.24
$(3 \mathrm{H}$, br. s, H Ad); 2.33 (6H, d, $J=2.8, \mathrm{H} \mathrm{Ad}) ; 2.55(3 \mathrm{H}, \mathrm{s}$, $\left.\mathrm{CH}_{3}\right) ; 7.33-7.43(3 \mathrm{H}, \mathrm{m}, \mathrm{H} \mathrm{Ar}) ; 7.90(1 \mathrm{H}, \mathrm{d}, J=7.6, \mathrm{H} \mathrm{Ar})$. ${ }^{13} \mathrm{C}$ NMR spectrum (DMSO- $\left.d_{6}\right), \delta$, ppm: $21.5\left(\mathrm{CH}_{3}\right) ; 29.3$, 35.6, 42.1, 64.0 (C Ad); 126.6, 126.9, 129.4, 130.4, 131.9, 137.1 (C Ar); 164.2 (C tetrazole). Found, $m / z$ : 295.1923 $[\mathrm{M}+\mathrm{H}]^{+} . \mathrm{C}_{18} \mathrm{H}_{23} \mathrm{~N}_{4}$. Calculated, $m / z: 295.1917$.

2-(Adamantan-1-yl)-5-(3-nitrophenyl)-2H-tetrazole (3c). Yield $2.52 \mathrm{~g}(86 \%)$, light-yellow crystals, mp 192 $193^{\circ} \mathrm{C}\left(\mathrm{mp} 192-193^{\circ} \mathrm{C}^{15}\right)$. ${ }^{1} \mathrm{H}$ NMR spectrum $\left(\mathrm{CDCl}_{3}\right)$, $\delta$, ppm $(J, \mathrm{~Hz}): 1.87$ (6H, br. s, H Ad); 2.35 (3H, br. s, H Ad); 2.43 (6H, br. s, H Ad); $7.70(1 \mathrm{H}, \mathrm{t}, J=8.0, \mathrm{H} \mathrm{Ar})$; 8.31-8.35 (1H, m, H Ar); 8.37 (1H, s, H Ar); 8.52-8.55 $(1 \mathrm{H}, \mathrm{m}, \mathrm{H} \mathrm{Ar}) .{ }^{13} \mathrm{C}$ NMR spectrum $\left(\mathrm{CDCl}_{3}\right), \delta$, ppm: 29.4 ; 35.8 ; 42.3; 64.5 (C Ad); 121.8, 124.1, 124.5, 127.6, 129.9, 132.4 (C Ar); 162.4 (C tetrazole). Found, $m / z: 348.0814$ $[\mathrm{M}+\mathrm{Na}]^{+} . \mathrm{C}_{17} \mathrm{H}_{19} \mathrm{~N}_{5} \mathrm{NaO}_{2}$. Calculated, $m / z: 348.1431$.

2-(Adamantan-1-yl)-5-(4-chlorophenyl)-2H-tetrazole (3d). Yield $2.38 \mathrm{~g}(84 \%)$, white crystals, $\mathrm{mp} 134-136^{\circ} \mathrm{C}$ (mp $\left.131-133^{\circ} \mathrm{C}^{13}\right) .{ }^{1} \mathrm{H}$ NMR spectrum $\left(\mathrm{CDCl}_{3}\right), \delta$, ppm $(J, \mathrm{~Hz}): 1.85$ (6H, t, $J=3.2, \mathrm{H} \mathrm{Ad}) ; 2.33$ (3H, br. s, H Ad); $2.41(6 \mathrm{H}, \mathrm{d}, J=2.8, \mathrm{H} \mathrm{Ad}) ; 7.47(2 \mathrm{H}, \mathrm{d}, J=8.8, \mathrm{H} \mathrm{Ar})$; $8.12(2 \mathrm{H}, \mathrm{d}, J=8.8, \mathrm{H} \mathrm{Ar}) .{ }^{13} \mathrm{C}$ NMR spectrum $\left(\mathrm{CDCl}_{3}\right)$, $\delta$, ppm: 29.4, 35.9, 42.3, 64.1 (C Ad); 126.5, 128.1 (2C), 129.0, 129.1, 135.9 (C Ar); 163.3 (C tetrazole). Found, $m / z$ : $315.1375[\mathrm{M}+\mathrm{H}]^{+} . \mathrm{C}_{17} \mathrm{H}_{20} \mathrm{ClN}_{4}$. Calculated, $m / z: 315.1371$.

4-[2-(Adamantan-1-yl)-2H-tetrazol-5-yl]-2-chloroaniline (3e). Yield $2.47 \mathrm{~g}$ (83\%), light-yellow crystals, mp $190-192^{\circ} \mathrm{C}$. IR spectrum, $v, \mathrm{~cm}^{-1}: 3202,2941,2916$, $1635,1606,1466,1420 .{ }^{1} \mathrm{H}$ NMR spectrum (DMSO- $d_{6}$ ), $\delta$, ppm $(J, \mathrm{~Hz}): 1.77(6 \mathrm{H}$, br. s, H Ad); $2.24(3 \mathrm{H}$, br. s, H Ad); 2.29 (6H, br. s, H Ad); 5.87 (2H, br. s, $\left.\mathrm{NH}_{2}\right), 6.91$ $(1 \mathrm{H}, \mathrm{d}, J=8.4, \mathrm{H} \mathrm{Ar}) ; 7.70(1 \mathrm{H}, \mathrm{d}, J=8.4, \mathrm{H} \mathrm{Ar}) ; 7.82(1 \mathrm{H}$, $\mathrm{d}, J=1.6, \mathrm{H} \mathrm{Ar}) .{ }^{13} \mathrm{C}$ NMR spectrum (DMSO- $\left.d_{6}\right), \delta$, ppm: 29.3, 35.7, 42.1, 63.8 (C Ad); 115.9, 117.4, 126.5, 127.4, 131.0, 147.1 (C Ar); 163.5 (C tetrazole). Found, $\mathrm{m} / \mathrm{z}$ : $330.1496[\mathrm{M}+\mathrm{H}]^{+} . \mathrm{C}_{17} \mathrm{H}_{21} \mathrm{ClN}_{5}$. Calculated, $m / z: 330.1480$.

2-(Adamantan-1-yl)-5-[2-chloro-4-(trifluoromethyl)phenyl]-2H-tetrazole (3f). Yield $2.79 \mathrm{~g}$ (81\%), white crystals, mp $126-128^{\circ} \mathrm{C} .{ }^{1} \mathrm{H}$ NMR spectrum (DMSO- $d_{6}$ ), $\delta$, ppm $(J, \mathrm{~Hz}): 1.76(6 \mathrm{H}$, br. s, H Ad); $2.24(3 \mathrm{H}$, br. s, H Ad); $2.33(6 \mathrm{H}, \mathrm{d}, J=2.8, \mathrm{H} \mathrm{Ad}) ; 7.89-7.92(1 \mathrm{H}, \mathrm{m}$, $\mathrm{H} \mathrm{Ar}) ; 8.08(1 \mathrm{H}, \mathrm{s}, \mathrm{H} \mathrm{Ar}) ; 8.15(1 \mathrm{H}, \mathrm{d}, J=8.0, \mathrm{H} \mathrm{Ar})$. ${ }^{13} \mathrm{C}$ NMR spectrum (DMSO- $\left.d_{6}\right), \delta$, ppm: $29.3,35.5,42.1$, 64.8 (C Ad); 125.0 (в, $\left.J=270.4, \mathrm{CF}_{3}\right) ; 125.8,128.1,130.6$, 132.8 (2C), 133.2 (C Ar); 161.1 (C tetrazole). Found, $m / z$ : $383.1252 \quad[\mathrm{M}+\mathrm{H}]^{+} . \quad \mathrm{C}_{18} \mathrm{H}_{19} \mathrm{ClF}_{3} \mathrm{~N}_{4} . \quad$ Calculated, $\quad \mathrm{m} / z$ : 383.1245 .

4-[(2-Adamantan-1-yl)-2H-tetrazol-5-yl]-2-nitrophenol (3g). Yield 2.64 g (86\%), light-orange crystals, mp 256$258^{\circ} \mathrm{C}$. ${ }^{1} \mathrm{H}$ NMR spectrum (DMSO- $\left.d_{6}\right), \delta$, ppm $(J, \mathrm{~Hz}): 1.79$ $(6 \mathrm{H}$, br. s, H Ad); 2.26 (3H, br. s, H Ad); 2.33 (6H, d, $J=2.4, \mathrm{H} \mathrm{Ad}) ; 7.33(1 \mathrm{H}, \mathrm{d}, J=8.4, \mathrm{H} \mathrm{Ar}) ; 8.19(1 \mathrm{H}, \mathrm{dd}$, $J=8.8, J=2.0, \mathrm{H} \mathrm{Ar}) ; 8.48(1 \mathrm{H}, \mathrm{d}, J=2.0, \mathrm{H} \mathrm{Ar}) ; 11.63$ $(1 \mathrm{H}, \mathrm{s}, \mathrm{OH}) .{ }^{13} \mathrm{C}$ NMR spectrum (DMSO- $\left.d_{6}\right), \delta, \mathrm{ppm}: 29.3$, 35.6, 42.1, 64.4 (C Ad); 105.0, 120.3, 123.6, 133.1, 139.6, 154.0 (C Ar); 164.9 (C tetrazole). Found, $m / z: 340.1400$ $[\mathrm{M}-\mathrm{H}]^{-} . \mathrm{C}_{17} \mathrm{H}_{18} \mathrm{~N}_{5} \mathrm{O}_{3}$. Calculated, $m / z: 340.1415$.

2-[2-(Adamantan-1-yl)-2H-tetrazol-5-yl]-6-bromo4-nitroaniline (3h). Yield $3.13 \mathrm{~g}$ (83\%), light-yellow 
crystals, mp $172-174^{\circ} \mathrm{C}$. IR spectrum, $v, \mathrm{~cm}^{-1}: 3346,2908$, $1611,1594,1495,1323,1303,1273,1142,1020 .{ }^{1} \mathrm{H}$ NMR spectrum $\left(\mathrm{CDCl}_{3}\right), \delta$, ppm $(J, \mathrm{~Hz}): 2.16(6 \mathrm{H}$, br. s, H Ad); $2.36(3 \mathrm{H}$, br. s, H Ad); $2.43(6 \mathrm{H}$, br. s, H Ad); $8.47(1 \mathrm{H}, \mathrm{d}$, $J=2.4, \mathrm{H} \mathrm{Ar}) ; 9.07(1 \mathrm{H}, \mathrm{d}, J=2.8, \mathrm{H} \mathrm{Ar}) .{ }^{13} \mathrm{C}$ NMR spectrum $\left(\mathrm{CDCl}_{3}\right), \delta$, ppm: 29.4, 36.1, 42.3, 64.9 (C Ad); 109.0, 109.9, 124.3, 124.5, 129.4, 148.2 (C Ar); 162.1 (C tetrazole). Found, $m / z$ : $417.0677[\mathrm{M}-\mathrm{H}]^{-} . \mathrm{C}_{17} \mathrm{H}_{18} \mathrm{BrN}_{6} \mathrm{O}_{2}$. Calculated, $\mathrm{m} / z: 417.0680$.

4-[2-(Adamantan-1-yl)-2H-tetrazol-5-yl]-2-aminophenol (3i). $\mathrm{HCl}$ and metallic $\mathrm{Zn}$ powder in small portions were successively added to a suspension of 4-[(2-adamantan1-yl)-2H-tetrazol-5-yl]-2-nitrophenol $(\mathbf{3 g})(2 \mathrm{mmol})$ in a mixture of $i-\mathrm{PrOH}(30 \mathrm{ml})$ and $\mathrm{H}_{2} \mathrm{O}(50 \mathrm{ml})$. The reaction mixture was heated to $50^{\circ} \mathrm{C}$ and maintained until the disappearance of the starting compound (TLC control, eluent $\mathrm{CHCl}_{3}-\mathrm{MeOH}, 19: 1$ ). The reaction mixture was then evaporated under reduced pressure. The residue was neutralized with an aqueous $\mathrm{NaOAc}$ to $\mathrm{pH} 4-5$. The formed precipitate was filtered off, air-dried, and recrystallized from $\mathrm{MeOH}$. Yield $2.30 \mathrm{~g}(82 \%)$, light-brown crystals, mp $157-159^{\circ} \mathrm{C} .{ }^{1} \mathrm{H}$ NMR spectrum (DMSO- $d_{6}$ ), $\delta$, ppm $(J, \mathrm{~Hz}): 1.78$ (6H, br. s, H Ad); 2.25 (3H, br. s, H Ad); 2.30 $2.34(6 \mathrm{H}, \mathrm{m}, \mathrm{H} \mathrm{Ad}) ; 4.77\left(2 \mathrm{H}\right.$, br. s, $\left.\mathrm{NH}_{2}\right) ; 6.76(1 \mathrm{H}, \mathrm{d}$, $J=8.4$, H Ar); 7.15 (1H, dd, $J=6.0, J=2.0, \mathrm{H} \mathrm{Ar}) ; 7.34$ $(1 \mathrm{H}, \mathrm{d}, J=2.0, \mathrm{H} \mathrm{Ar}) .{ }^{13} \mathrm{C}$ NMR spectrum (DMSO- $d_{6}$ ), $\delta$, ppm: 29.3, 35.6, 42.1, 64.4 (C Ad); 107.5, 123.7, 128.1, 133.1, 137.5, 150.8 (C Ar); 162.5 (C tetrazole). Found, $m / z: 312.1814 \quad[\mathrm{M}+\mathrm{H}]^{+} . \quad \mathrm{C}_{17} \mathrm{H}_{22} \mathrm{~N}_{5} \mathrm{O}$. Calculated, $m / z$ : 312.1819 .

Nitration of 5-aryltetrazole adamantyl derivatives (General method). A mixture of concentrated $\mathrm{HNO}_{3}(7 \mathrm{ml})$ and concentrated $\mathrm{H}_{2} \mathrm{SO}_{4}(5 \mathrm{ml})$ was added to a cooled $\left(5^{\circ} \mathrm{C}\right)$ solution of adamantyl derivative of tetrazole $\mathbf{3 c}, \mathbf{d}(6 \mathrm{mmol})$ in $75 \% \mathrm{H}_{2} \mathrm{SO}_{4}(7 \mathrm{ml})$. The reaction mixture was stirred at $8-10^{\circ} \mathrm{C}$ for $3 \mathrm{~h}$, then the $\mathrm{pH}$ of the reaction mixture was adjusted to 7 by adding an aqueous $\mathrm{NaOH}$. The precipitated product was filtered off and dried.

2-(Adamantan-1-yl)-5-(3,5-dinitrophenyl)-2 $\mathrm{H}$-tetrazole (3j). Yield $1.84 \mathrm{~g}(85 \%)$, white crystals, $\mathrm{mp} 276-277^{\circ} \mathrm{C}$ (mp 276-277 $\left.{ }^{\circ} \mathrm{C}^{15}\right) .{ }^{1} \mathrm{H}$ NMR spectrum $\left(\mathrm{CDCl}_{3}\right), \delta$, ppm $(J, \mathrm{~Hz}): 1.75-1.85$ (6H, m, H Ad); 2.29 (3H, br. s, H Ad); 2.39 (6H, br. s, H Ad); 8.96 (1H, t, $J=2.1, \mathrm{H} \mathrm{Ar}) ; 9.07$ $(2 \mathrm{H}, \mathrm{d}, J=2.1, \mathrm{H} \mathrm{Ar}) .{ }^{13} \mathrm{C}$ NMR spectrum $\left(\mathrm{CDCl}_{3}\right)$, $\delta$, ppm: 29.3, 35.5, 45.7, 65.2 (C Ad); 120.3, 126.5, 130.3, 149.3 (C Ar); 161.1 (C tetrazole). Found, $m / z: 393.1250$ $[\mathrm{M}+\mathrm{Na}]^{+}$. $\mathrm{C}_{17} \mathrm{H}_{17} \mathrm{~N}_{6} \mathrm{NaO}_{4}$. Calculated, $m / z$ : 393.1282 .

2-(Adamantan-1-yl)-5-(4-chloro-3-nitrophenyl)-2Htetrazole (3k). Yield $1.95 \mathrm{~g}(88 \%)$, white crystals, mp 149$151{ }^{\circ} \mathrm{C} .{ }^{1} \mathrm{H}$ NMR spectrum (DMSO- $\left.d_{6}\right), \delta$, ppm $(J, \mathrm{~Hz})$ : $1.52-1.80$ (6H, m, H Ad); $2.18-2.45$ (3H, m, H Ad); 2.33 $(6 \mathrm{H}$, br. s, H Ad); 7.97 (1H, d, $J=8.4, \mathrm{H} \mathrm{Ar}) ; 8.33(1 \mathrm{H}, \mathrm{dd}$, $J=8.4, J=2.0, \mathrm{H} \mathrm{Ar}) ; 8.63(1 \mathrm{H}, \mathrm{d}, J=2.0, \mathrm{H} \mathrm{Ar})$. ${ }^{13} \mathrm{C}$ NMR spectrum (DMSO- $d_{6}$ ), $\delta$, ppm: $29.3,35.6,42.1$, 64.9 (C Ad); 105.0, 114.8, 123.6, 131.7, 133.4, 148.5 (C Ar); 162.9 (C tetrazole). Found, $m / z: 360.1225[\mathrm{M}+\mathrm{H}]^{+}$. $\mathrm{C}_{17} \mathrm{H}_{19} \mathrm{ClN}_{5} \mathrm{O}_{2}$. Calculated, $\mathrm{m} / z: 360.1222$.

The study of the biological activity of compounds $\mathbf{3 a}, \mathbf{b}, \mathbf{d}, \mathbf{e}, \mathbf{h}, \mathbf{i}$. The cytotoxic properties of compounds were assessed using the methyl tetrazolium (MTT) assay. A series of threefold dilutions $(300-3.7 \mu \mathrm{g} / \mathrm{ml})$ were prepared from the studied compounds, after which they were introduced into the wells of plates with a monolayer of MDCK cells. The plates were incubated at $36^{\circ} \mathrm{C}$ for $72 \mathrm{~h}$. Thereafter, MTT solution was added to the wells which by the action of mitochondrial enzymes transformed into an insoluble violet formazan derivative. ${ }^{18}$ Plates with cells were kept for $2 \mathrm{~h}$. The sediment in each well was dissolved in DMSO $(0.1 \mathrm{ml})$. The optical density in the wells was measured using a Thermo Multiskan FC plate photometer (Thermo Fisher Scientific, USA) with a wavelength of $540 \mathrm{~nm}$. Based on the obtained data, the $\mathrm{CC}_{50}$ value was calculated as the concentration of the compound resulting in a decrease in optical density by half compared to the wells without addition of compounds.

The study of the antiviral activity of compounds $\mathbf{3 a}, \mathbf{b}, \mathbf{d}, \mathbf{e}, \mathbf{h}, \mathbf{i}$ was carried by determining the reduction of the degree of cytopathic effect. The experiments used the influenza A/Puerto Rico/8/34 (H1N1) virus. The studied compounds in various concentrations were added to the cells in the wells of the plate, incubated for $1 \mathrm{~h}$, then the cells were infected with the virus at a dose of $0.01 \mathrm{TCID}_{50}$ per cell. The cells were incubated for $72 \mathrm{~h}$, after which the cell viability was determined by the MTT assay as described above. Based on the obtained data for each compound, $50 \%$ inhibitory concentration $\left(\mathrm{IC}_{50}\right)$ was calculated as the concentration of the compound that reduces the degree of viral cell destruction by $50 \%$, and the selectivity index (SI) as the ratio of $\mathrm{CC}_{50}$ to $\mathrm{IC}_{50}$.

$\mathrm{X}$-ray structural analysis of compounds $3 \mathrm{~b}, \mathrm{e}-\mathrm{g}$. Crystals suitable for X-ray structural analysis were obtained by slow evaporation of solutions of compounds 3b,e- $\mathbf{g}$ in $\mathrm{CHCl}_{3}-\mathrm{MeOH}, 1: 2$ mixture at low temperature.

X-ray structural analysis of compounds $\mathbf{3 b}, \mathbf{e}-\mathbf{g}$ was done on a Rigaku Oxford Diffraction XtaLAB SuperNova diffractometer with a HyPix3000 CCD detector (compounds 3b,e,f) and on an Agilent Technologies Xcalibur diffractometer with an Atlas CCD detector (compound 3g) at $100 \mathrm{~K}$ using monochromatic focused $\mathrm{CuK} \alpha$ radiation. The obtained data were integrated in the CrysAlis software package. ${ }^{19}$ Absorption was corrected semiempirically. The signs of disordering of the adamantane group are removed from Figure 3 (compound 3e) for the sake of clarity. The unit cell parameters were specified by the least-squares method. The structures were solved and refined using the SHELX software package ${ }^{20}$ included in the OLEX2 ${ }^{21}$ interface. Crystallographic data of compounds $\mathbf{3 b}, \mathbf{e}-\mathbf{g}$ were deposited at the Cambridge Crystallographic Data Center (deposits CCDC 1984233 (compound 3b), CCDC 1984234 (compound 3e), CCDC 1984236 (compound 3f), CCDC 1984237 (compound 3g)).

Supplementary information file containing the IR, ${ }^{1} \mathrm{H}$ and ${ }^{13} \mathrm{C}$ NMR, and mass spectra of all the synthesized compounds, simultaneous thermal analysis data for compound $\mathbf{3 g}$, as well as X-ray structural analysis data for compounds $\mathbf{3 b}, \mathbf{e}-\mathbf{g}$ is available at the journal website at http://link.springer.com/journal/10593. 
This work was financially supported by the Russian Foundation for Basic Research (project 20-53-00039Bel_a).

The work was carried out using the equipment of the resource centers of Saint Petersburg State University "Magnetic Resonance Research Center", "Chemical Analysis and Materials Research Center", "Chemistry Educational Center".

\section{References}

1. Stockdale, T. P.; Williams, C. M. Chem. Soc. Rev. 2015, 44, 7737.

2. Suslov, E. V.; Mozhaytsev, E. S.; Korchagina, D. V.; Bormotov, N. I.; Yarovaya, O. I.; Volcho, K. P.; Serova, O. A.; Agafonov, A. P.; Maksyutov, R. A.; Shishkina, L. N.; Salakhutdinov, N. F. RSC Med. Chem. 2020, 11, 1185.

3. Pham, V. H.; Phan, T. P. D.; Phan, D. C.; Vu, B. D. Molecules 2020, 25, 324.

4. Kreutzberger, A.; Schröders, H.-H.; Stratmann, J. Arch. Pharm. 1984, 317, 767.

5. Kreutzberger, A.; Schröders, H.-H. Arch. Pharm. 1974, 307, 766.

6. Parsons, C. G.; Gruner, R.; Rozental, J.; Millar, J.; Lodge, D. Neuropharmacology 1993, 32, 1337.

7. Rejdak, K.; Grieb, P. Mult. Scler. Relat. Disord. 2020, 42, 102163.

8. Cimolai, N. J. Med. Virol. 2020, 92, 531.

9. Ostrovskii, V. A.; Trifonov, R. E.; Popova, E. A. Russ. Chem. Bull., Int. Ed. 2012, 61, 768. [Izv. Akad. Sci., Ser. Khim. 2012, 765.]

10. Popova, E. A.; Trifonov, R. E.; Ostrovskii, V. A. Russ. Chem. Rev. 2019, 88, 644. [Usp. Khim. 2019, 88, 644.]
11. Ostrovskii, V. A.; Popova, E. A.; Trifonov, R. E. Adv. Heterocycl. Chem. 2017, 123, 1.

12. Zarubaev, V. V.; Golod, E. L.; Anfimov, P. M.; Shtro, A. A.; Saraev, V. V.; Gavrilov, A. S.; Logvinov, A. V.; Kiselev, O. I. Bioorg. Med. Chem. 2010, 18, 839.

13. Seliverstova, D. V.; Suslonov, V. V.; Zarubaev V. V.; Trifonov R. E. Russ. J. Org. Chem. 2018, 54, 633. [Zh. Org. Khim. 2018, 54, 630.]

14. Ostrovskii, V. A.; Koldobskii, G. I.; Trifonov, R. E. In Comprehensive Heterocyclic Chemistry III; Zhdankin, V. V., Ed.; Elsevier: Amsterdam, 2008, Vol. 6, p. 257.

15. Mikolaichuk, O. V.; Spasibenko, D. V.; Trifonov, R. E. Chem. Heterocycl. Compd. 2020, 56, 961. [Khim. Geterotsikl. Soedin. 56, 961.]

16. D'yachenko, V. S.; Danilov, D. V.; Shkineva, T. K.; Vatsadze, I. A.; Burmistrov, V. V.; Butov, G. M. Chem. Heterocycl. Compd. 2019, 55, 129. [Khim. Geterotsikl. Soedin. 2019, 55, 129.]

17. Mikolaichuk, O. V.; Batyrenko A. A.; Kornyakov I. V.; Trifonov, R. E. J. Heterocycl. Chem. 2021, DOI: doi/10.1002/ jhet.4232.

18. Mosmann, T. J. Immunol. Methods 1983, 65, 55.

19. CrysAlisPro Software system, version 1.171.39.50a; Rigaku Oxford Diffraction: Oxford, 2019.

20. (a) Sheldrick, G. M. Acta Crystallogr., Sect. A: Found. Adv. 2015, A71, 3. (b) Sheldrick, G. M. Acta Crystallogr., Sect. C: Struct. Chem. 2015, C71, 3.

21. Dolomanov, O. V.; Bourhis, L. J.; Gildea, R. J.; Howard, J. A. K.; Puschmann, H. J. Appl. Crystallogr. 2009, 42, 339. 